\title{
Testosterone is Key to Increase the Muscle Capillary Density of Old and Trained Rats
}

\author{
Walter Krause Neto ${ }^{1}$ Wellington de Assis Silva ${ }^{1}$ Amanda dos Santos da Silva ${ }^{1}$ Adriano Polican Ciena ${ }^{2}$ \\ Romeu Rodrigues de Souza ${ }^{1}$ Patricia Oliva Carbone ${ }^{1}$ Carlos Alberto Anaruma ${ }^{2}$ Eliane Florencio Gama ${ }^{1}$ \\ 1 Department of Physical Education, Laboratory of \\ Morphoquantitative Studies and Immunohistochemistry, \\ Universidade São Judas Tadeu, São Paulo, SP, Brazil \\ ${ }^{2}$ Department of Physical Education, Laboratory of Morphology and \\ Address for correspondence Walter Krause Neto, PhD, Rua Taquari, \\ 546, Mooca, 03166-000, São Paulo, SP - Brazil \\ (e-mail: wild_krause@hotmail.com).
} Physical Activity, Universidade Estadual Paulista “Júlio de Mesquita Filho", Rio Claro, SP, Brazil

J Morphol Sci 2019;36:182-189.

\author{
Abstract \\ Keywords \\ - aging \\ - blood vessels \\ - skeletal muscle \\ - training \\ - steroids
}

Introduction It is indicated that aging reduces muscular vascularity. Thus, strategies that aim to reverse or decrease the progression of these changes are suggested. Therefore, the present study aimed to analyze the blood capillary density of the plantaris muscle (PL) of aged rats submitted to strength training and exogenous testosterone use.

Methods A total of 30 rats were divided equally into the following groups: EA: 20 months old; OLD: 24 months old; OLD + S: testosterone; ST: strength training; and ST + S: strength training + testosterone. Strength training was performed 3 times a week for 15 weeks. Testosterone administration was performed by the intraperitoneal method 2 times a week at a dose of $10 \mathrm{mg} / \mathrm{kg}$. All of the morphological parameters were analyzed using morphoquantitative techniques.

Results A significant increase in the capillaries numerical density of the OLD $+S$ and $\mathrm{ST}+\mathrm{S}$ groups. The OLD $+\mathrm{S}$ and ST $+\mathrm{S}$ groups demonstrated a higher ratio of capillaries per myofiber than the EA, OLD and ST groups. The OLD and ST groups had a higher ratio than the EA group. The OLD $+S$, ST and ST + S groups presented a statistically significant increase in the cross-sectional area in the type I fibers when compared with the EA and OLD groups. However, the ST group showed a significant increase compared with the OLD group. In type ll fibers, the ST and ST + S groups showed a greater cross-sectional area than the EA group. As for the mean cross-sectional area, the ST and ST $+\mathrm{S}$ groups presented significantly larger areas than the other groups.

Conclusion Anabolic steroid administration is crucial to increase the blood capillary density of the plantaris muscle of old rats, independently of the effects of strength training.

\section{Introduction}

Age advance induces morphological and physiological changes that can be noticed and understood over time in various biological systems. Among the changes that occur in the neuromuscular system, we can mention sarcopenia (reduction of muscle mass) and dynapenia (loss of muscle strength) ${ }^{1,2}$ In addition, it is possible to note changes that also affect the vascular system, ${ }^{3,4}$ where the loss of muscle mass over time causes a decrease in blood capillary density, compromising the transport system and altering important biochemical processes responsible for maintaining muscular endurance. Thus, plenty of evidence seek to bring received

March 11, 2019

accepted

May 1, 2019
DOI https://doi.org/

10.1055/s-0039-1692209. ISSN 2177-0298.
Copyright $\odot 2019$ by Thieme Revinter

Publicações Ltda, Rio de Janeiro, Brazil
License terms

(c) $(1) \$$ 
strategies to minimize the effects associated with biological aging. 5,6

In an attempt to delay or mitigate these changes, physical training has been cited as a fundamental strategy to maintain functional capacity. ${ }^{7}$ Aerobic training is an excellent strategy for the cardiovascular adaptations, since, over time, blood vessels lose elasticity, capillary diameter, and endothelial function, and also present a reduced blood capillaries/muscle fiber ratio. ${ }^{8-10}$ However, strength training also provides benefits, such as increased muscle mass, concomitantly with muscular strength. However, little is known about adaptations that may occur in the vascular system from muscles submitted to strength training. Recently, Krause Neto et al ${ }^{11}$ have shown that vascularization of different types of skeletal muscles in middle-aged rats may undergo inversely proportional adjustments. According to Hudlicka et al, ${ }^{12}$ this controversial information can be linked to the stimulus that each type of exercise can make possible the different particularities of muscle fibers.

Studies show that the hormone testosterone has an important impact in the vascular system functions, such as a reduction in the proportion of atheroma plaques in the aorta, indicating a direct action of the hormone in the endothelial tissue. ${ }^{13,14}$ When combined with strength training, testosterone may present a potent impact over the cardiovascular system, by increasing the proportion of capillaries in the heart of rodents. ${ }^{15}$ Therefore, if aging can lower the ratio of capillaries to myofibers as previously mentioned, strength training in conjunction with testosterone may become an alternative to avoid decreasing blood capillary density in old age.

Therefore, the aim of the present study was to analyze the blood capillary density of the plantaris muscle of aged Wistar rats submitted to strength training associated with exogenous testosterone.

\section{Materials and Methods}

These procedures were authorized by the Committee on Ethics in Animal Use (Protocol 001/2013) of the Universidade São Judas Tadeu (USJT, in the Portuguese acronym).

\section{Division of Animals}

Wistar rats (rattus norvegicus), aged 20 months, were divided into the following groups: EA: control at 20 months old $(n=6)$; OLD: control at 24 months old $(n=6)$; OLD + S: control at 20 months old who received administration of exogenous testosterone $(n=6)$; ST; animals submitted to strength training (ST) protocol $(n=6) ; \mathrm{ST}+\mathrm{S}$ : animals submitted to strength training protocol in conjunction with exogenous testosterone administration $(n=6)$.

During the experiment, the animals were kept in polypropylene boxes (a maximum of three animals each) provided with a drinking fountain and a feeder, kept under controlled conditions of temperature $\left(22^{\circ} \mathrm{C}\right)$ and illumination (12 hours of light and 12 hours of darkness). All of the rats were fed commercial reference feed for rats and water ad libitum.

\section{Strength Training}

The ST protocol was performed during the active period of the animal (nocturnal), by reversing the light-dark cycle of the experiment room. The ladder climbing (LC) equipment was built in wood with iron steps, $110 \mathrm{~cm}$ in height, $80^{\circ}$ of inclination, and with a distance of $2 \mathrm{~cm}$ between each step.

To adapt the animals to the equipment, each rodent climbed the ladder three times, starting from three distinct positions (upper third, middle, and base). The animals had no aid or reward to climb the equipment, taking a minute interval between each climb. If necessary, a touch was given on the tail of the rodent to start the climbing movement. This process was conducted for five sessions, Monday through Friday, without any additional burden attached to the tail of the rodent. After this period, the animals of all of the groups underwent the maximum carrying load test (MCLT) to determine the training loads.

The MCLT was performed according to the one proposed by Deus et al $^{16}$ and adapted by Krause Neto et al. ${ }^{17}$ In this case, each animal had to climb the ladder as many times as possible from a given initial load, adding a new percentage to each attempt. Thus, the protocol was conducted as follows: (1) each animal was previously weighed and the relative loads were calculated; (2) each rodent climbed the ladder twice, without any additional load, with a 1 minute interval between each climb; (3) initial testing load calculated from $50 \%$ of the body weight (BW) of the animal and (4) subsequent increases of $10 \%$ of the BW for each successful climbing. The test was conducted until the animal was unable to climb the ladder for at least two consecutive trials (failure). Between each attempt, the animal was allowed 2 minutes of recovery.

After the MCLT, the ST and ST + S groups underwent an ST protocol 3 times a week for 15 weeks ( 45 sessions). For this, we used the protocol previously described by Harris et al. ${ }^{18}$ In the first training session, the rats climbed the ladder twice with the following loads: 50, 75 and $100 \%$ MCLT. An increase of 30 grams was made from the $6^{\text {th }}$ consecutive climb if the animal could climb the ladder twice with $100 \%$. For this, the rodent was able to climb the ladder two more times, totaling, at the end, eight maximum climbs. In the subsequent sessions, the training load percentages (50, 75 and 100\%) were calculated from the new maximum load reached in the previous session. However, if the animal did not complete the protocol with eight maximum climbs, the same loads were maintained for the next training session. Between each climb, each rodent took an interval of 2 minutes.

For the increase of loads, we used lead weights attached by a musket and adhesive tape, tied to the proximal region of the tail of the animals ( $\mathbf{- F i g . 1} \mathbf{1}$ ).

\section{Anabolic Steroid}

For the present study, we used the PERINON anabolic steroid (Laboratório Perini, São José, SC, Brazil) in an ampoule bottle with $100 \mathrm{ml}$ containing $200 \mathrm{mg} / 20 \mathrm{ml}$ of testosterone propionate (TP). The dosage used was $10 \mathrm{mg} / \mathrm{kg} / \mathrm{BW} /$ week. Previous data from our laboratory demonstrated significant muscle hypertrophy and low level of hepatic side effect with 


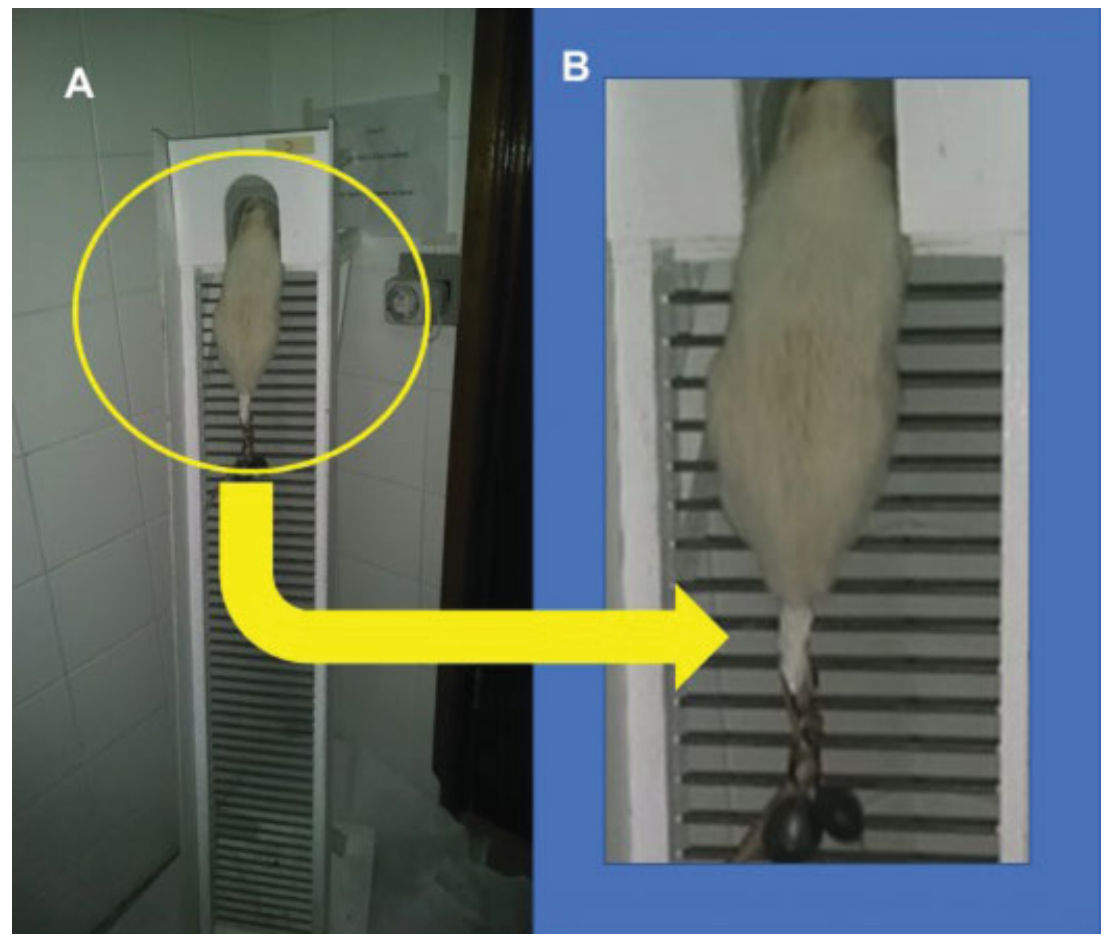

Fig. 1 Illustrative image of the ladder climbing equipment (A) with load attached to the tail (yellow arrow) of the rodent during the period of strength training (blue box, B).

this dosage. ${ }^{11,19}$ The animals were weighed every week to recalculate the dosage applied.

The administration of TP was performed by intraperitoneal injection, twice weekly (always on Tuesdays and Fridays), starting from the $1^{\text {st }}$ day of the experiment, in the $\mathrm{OLD}+\mathrm{S}$ and ST $+\mathrm{S}$ groups, during 15 weeks. The animals received the injections from the age of 20 months old, shortly after the MCLT, until the scheduled date for the euthanasia.

\section{Euthanasia, Collection and Preparation of the Material for Analysis}

The animals were euthanized by the $\mathrm{CO}_{2}$ inhalation method. After the euthanasia, we removed the plantaris muscle (PL), whose choice was due to the predominance of muscle fiber typology (type II) and action during the climbing exercise.

An incision in the posterior portion of the right knee of the animal up to the ankle was performed to expose the PL muscle, then it was cryopyzed in liquid nitrogen and kept at $-80^{\circ} \mathrm{C}$ until the analysis.

For the analysis of tissue quality and general morphology, $7 \mu \mathrm{m}$ of the tissue was sectioned in a cryostat at $-20^{\circ} \mathrm{C}$ and prepared with hematoxylin and eosin (H\&E) (-Fig. 2).

For the analysis of the typology of the muscle fiber, we used staining with Nicotinamide adenine dinucleotide tetrazolium reductase (NADH-TR) through tissue section in a cryostat at $-20^{\circ} \mathrm{C}$, incubation of the slides for 40 minutes at $37^{\circ} \mathrm{C}$ in a solution containing $8 \mathrm{mg}$ of NADH, $10 \mathrm{mg}$ of Nitro Blue Tetrazolium (NBT) and $0.2 \mathrm{M}$ of Tris buffer ( $\mathrm{pH}$ 7.4). The material was then washed in $30 \%, 60 \%$, and $90 \%$ increasing acetone p.a, then returned to $60 \%$ and $30 \%$. Finally, it was assembled into Jeli glycerin (-Fig. $\mathbf{3}$ ).

\section{Morphoquantitative Analysis}

The stereological study was applied using the physical dissector technique and the ImageJ software (National Institutes of Health, Bethesda, MD, USA). This program allowed us to estimate the numerical density of capillaries (NV[CAP]) and of muscle fibers (NV[MYOFIBERS]). We calculated the number of capillaries per frame, and the number of muscle fibers (excluding those touching the upper and left side of the image) and the ratio of capillaries per muscle fibers (RATIO [CAPILLARIES/MYOFIBER]) were calculated by dividing the total number of capillaries of the frame by the relative number of field muscle fibers. For this procedure, 20 images

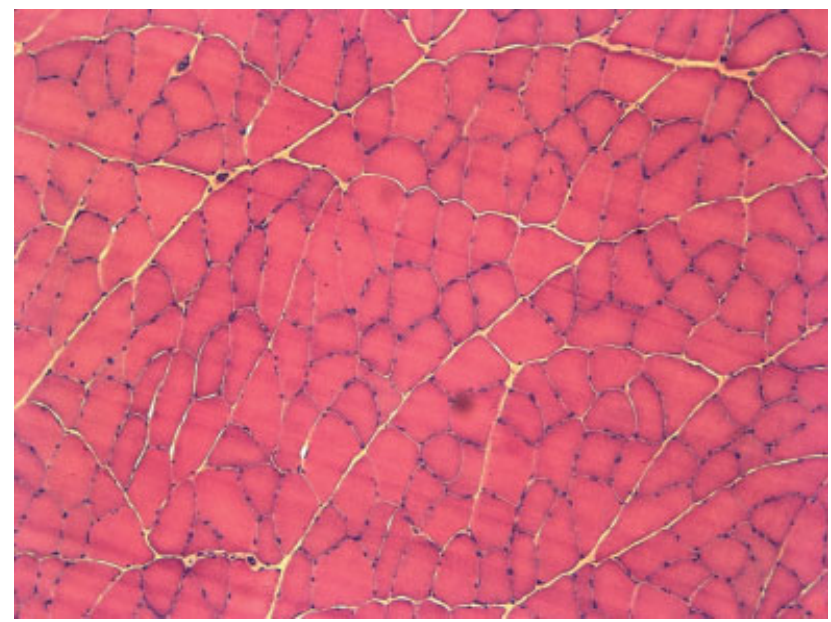

Fig. 2 Cross-sectional area of plantaris muscle stained with hematoxylin and eosin (H\&E). I 100x magnification. 


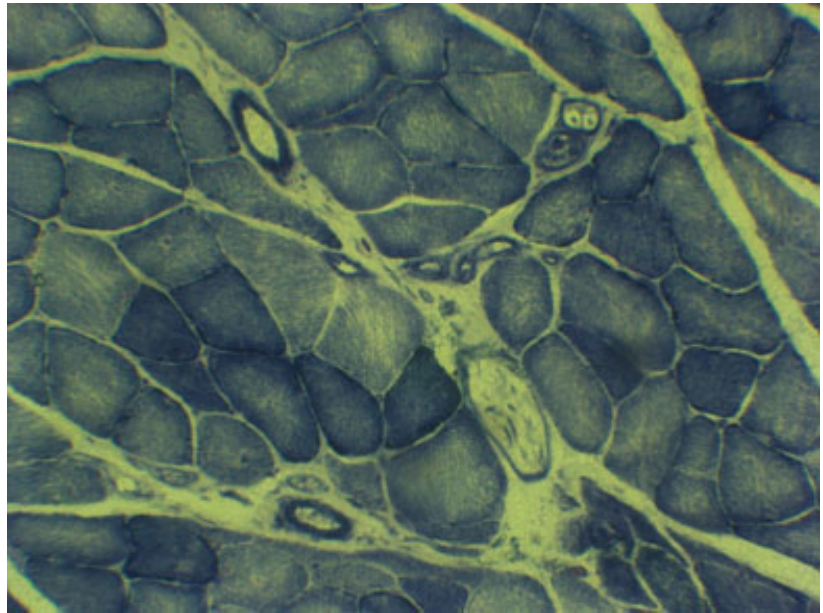

Fig. 3 Cross-sectional area of the plantaris muscle with Nicotinamide adenine dinucleotide tetrazolium reductase (NADH-TR) staining. 200x magnification.

of each group with a final magnification of 100x were used (-Fig. 4).

For the morphometry, 30 photographs of each group with a final magnification of 400x were analyzed. The measurements were made in the Axiovision software version 4.8 (Carl Zeiss, Oberkochen, Germany) coupled to a light microscope (Carl Zeiss, Oberkochen, Germany). In each slide, it was possible to measure the cross-sectional area $\left(\mu \mathrm{m}^{2}\right)$ of type I (oxidative) and II (glycolytic) muscle fibers (-Fig. 5).

\section{Statistical Analysis}

Data are presented by mean and standard deviation [SD]. For the statistical comparison between the different groups, two-way analysis of variance (ANOVA) (post hoc Tukey) was used. For statistical calculations, IBM SPSS Statistics for

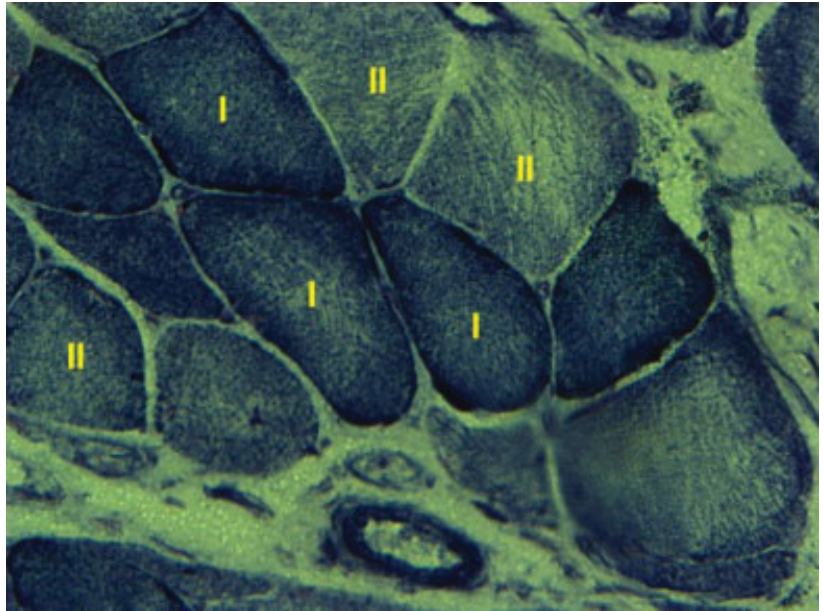

Fig. 5 Illustrative image of the Nicotinamide adenine dinucleotide tetrazolium reductase (NADH-TR) labeling of the type I (dark) and II (clear) myofibers of the plantaris muscle. 400x magnification.

Windows, Version 21.0 (IBM Corp., Armonk, NY, USA) was used and the level of significance was set at $p \leq 0.05$.

\section{Results}

The analysis of the numerical density of capillaries showed a significant difference between the groups $(\mathrm{F}=8.718$; $p=0.0001)$. It was observed that there was a statistically significant increase $(p \leq 0.05)$ in NV[CAP] of the OLD + S and $\mathrm{ST}+\mathrm{S}$ groups in relation to the EA, OLD and ST groups $(p<0.05)$, without any statistical difference between both groups(-Fig. 6).

In - Fig. 7, NV[MYOFIBERS] by field was statistically lower in the ST group compared with in the EA group $(p<0.05)$. ST + S group showed less muscle fibers per field than the EA and OLD groups $(p<0.05)$.

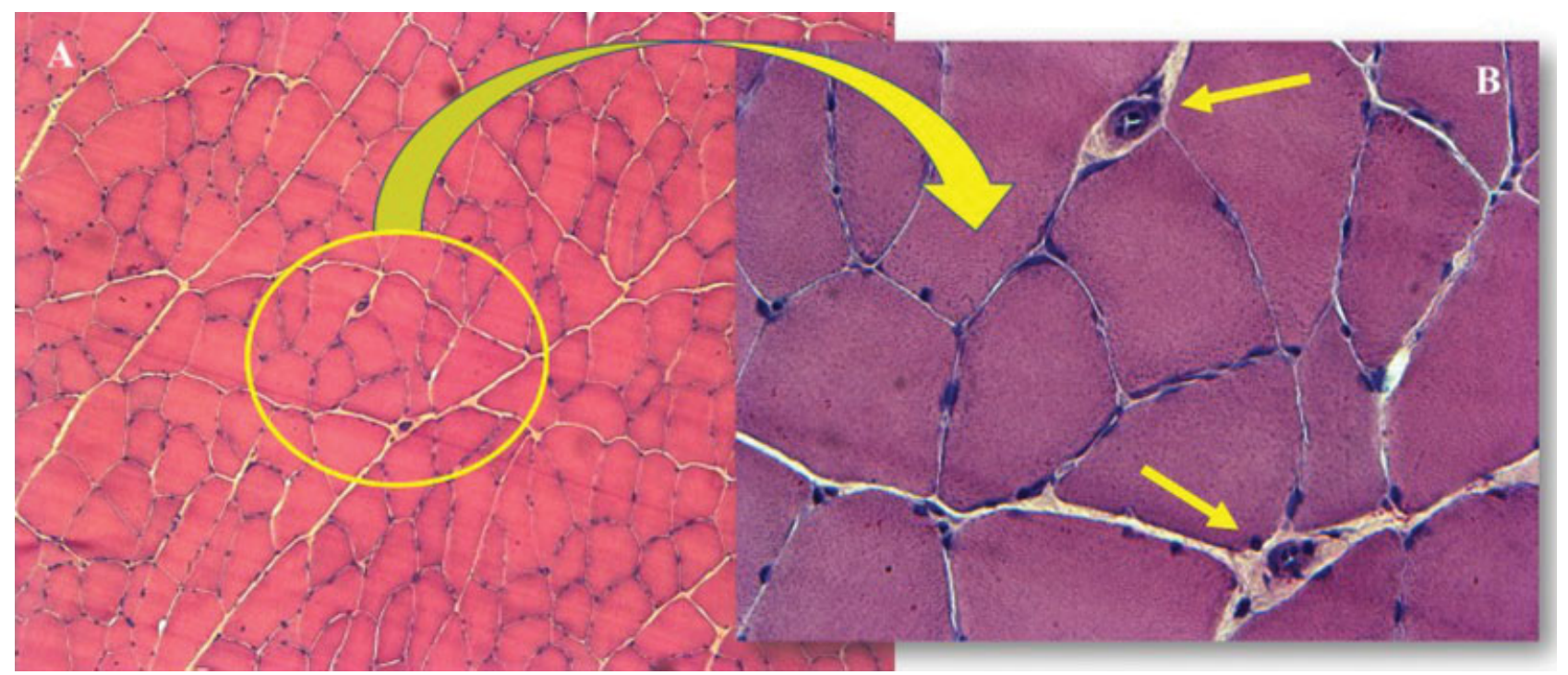

Fig. 4 Cross-sectional area of the plantaris muscle of Wistar rats with hematoxylin and eosin staining. (A) image with a magnification of 100x, used for the quantification of the numerical density of capillaries and muscle fibers; (B) image with magnification of $400 x$ to confirm the positioning of the blood capillaries (yellow arrows). 


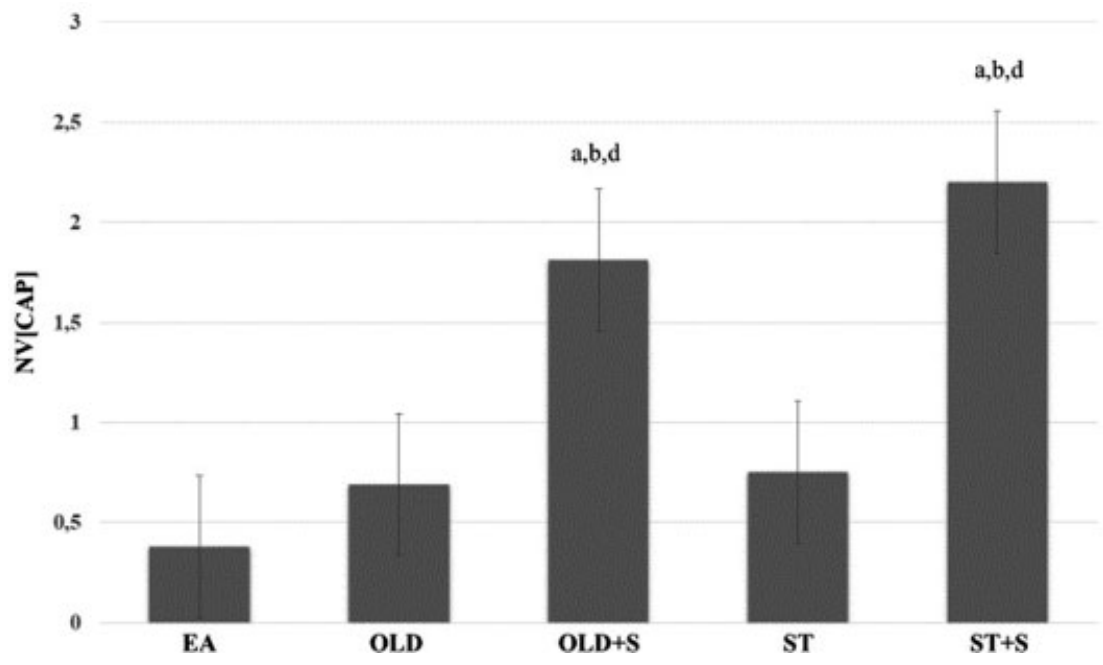

Fig. 6 Numerical density of capillaries (NV[CAP]) of the plantaris muscle of aged Wistar rats: 20 months of age (EA), 24 months of age (OLD), 24 months of age + steroid $(O L D+S)$, strength training (ST) and strength training + steroid $(S T+S)$. (a) represents significant difference versus EA; (b) represents significant difference vs OLD, (d) represents significant difference versus ST; $F=8.718 ; p=0.0001$.

Regarding the ratio [CAPILLARIES/MYOFIBER] (-Fig. 8), the $\mathrm{OLD}+\mathrm{S}$ and $\mathrm{ST}+\mathrm{S}$ groups demonstrated a higher number of capillaries by muscle fiber than the EA, OLD and ST groups $(\mathrm{F}=3.423 ; p=0.0001)$. Still, the OLD and ST groups had a higher ratio than the EA group $(\mathrm{p}<0.05)$.

The OLD + S, ST and ST + S groups presented a statistically significant increase $(p \leq 0.05)$ in the cross-sectional area in the type I fibers when compared with the EA and OLD groups $(\mathrm{F}=9.894 ; p=0.0001)$. The ST group showed a significant increase compared with the OLD group $(p<0.05)$. In type II fibers, the ST and ST $+\mathrm{S}$ groups showed a greater cross-sectional area than the $\mathrm{AE}(\mathrm{F}=4.817$; $p=0.001$ ). As for the mean cross-sectional area (-Fig. 9), those of the ST and ST $+\mathrm{S}$ groups were significantly larger than those of the other groups $(\mathrm{F}=7.832 ; \mathrm{p}=0.0001)$.

\section{Discussion}

Our study presented some interesting results: (1) there was no significant reduction of blood capillary density among the aged control groups, despite the higher ratio of capillaries/ myofibers in the plantaris muscle; (2) testosterone administration influenced morphological response (NV[CAP], ratio [CAPILLARIES/MYOFIBER] and muscle hypertrophy) in all of the groups, with and without ST.

In relation to aging, our results demonstrate a minimal and not significant morphological alteration, which is in line with other studies already published in the literature. ${ }^{20,21}$ Muscle blood capillarity is determined partly by the muscle size and metabolic profile, then by the predominance of muscle fiber type. ${ }^{22,23}$ Thus, the muscular characteristics

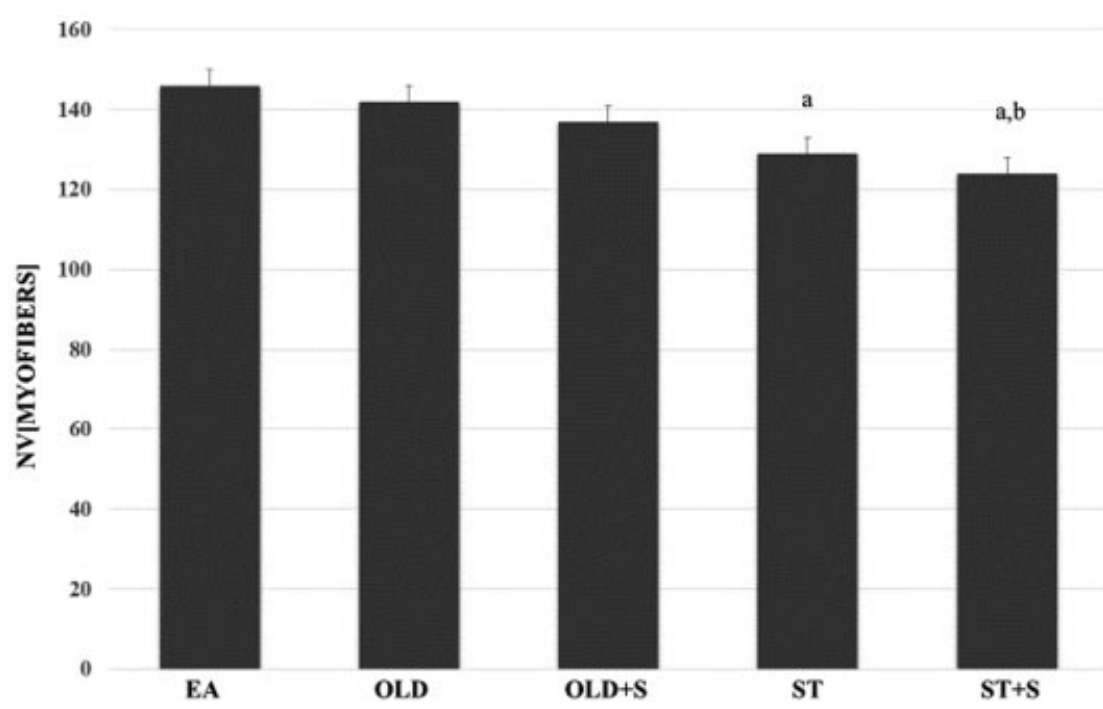

Fig. 7 Numerical density of myofibers (NV[MYOFIBERS]) per field of plantaris muscle of aged Wistar rats: 20 months old (EA), 24 months old (OLD), 24 months old + steroid (OLD $+S$ ), strength training (ST), and strength training + steroid $(S T+S)$. (a) represents significant difference versus EA; (b) represents significant difference versus OLD; $\mathrm{F}=6.974 ; p=0.002$. 


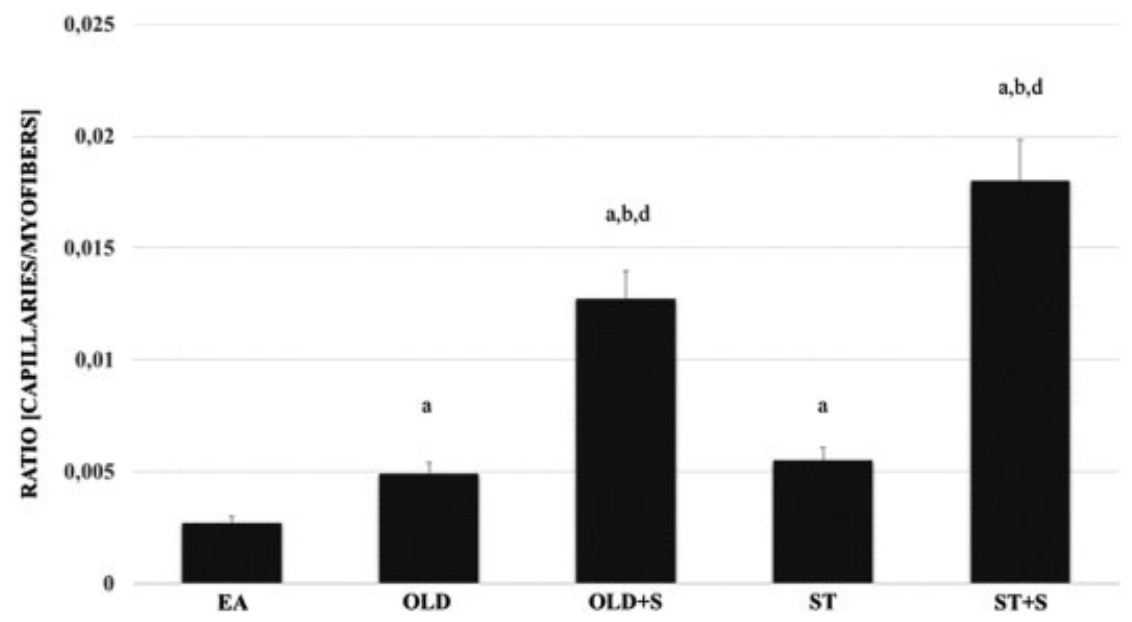

Fig. 8 Ratio of capillaries per muscle fibers (RATIO[CAPILLARIES/MYOFIBERS]) of the plantaris muscle of aged Wistar rats: 20 months old (EA), 24 months old (OLD), 24 months old + steroid (OLD + S), strength training (ST), and strength training + steroid (ST + S). (a) represents significant difference versus EA; (b) represents significant difference versus OLD, (d) represents significant difference versus ST; $F=12.007$; $p=0.0001$.

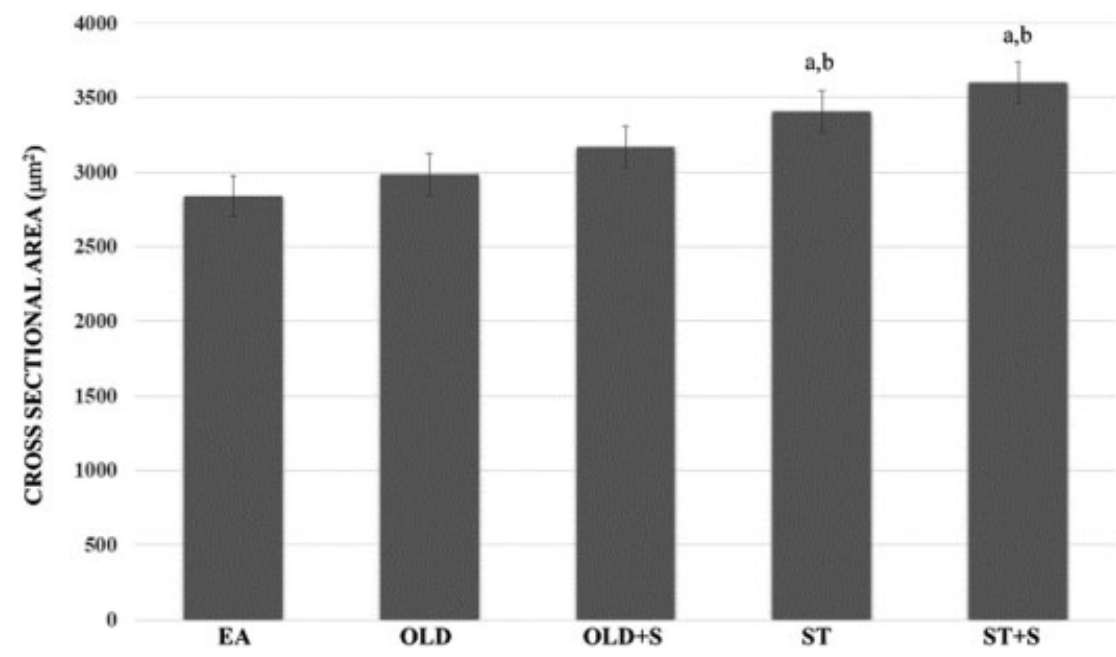

Fig. 9 Mean cross-sectional area $\left(\mu \mathrm{m}^{2}\right)$ of the plantaris muscle of aged Wistar rats: 20 months old (EA), 24 months old (OLD), 24 months old + steroid $(O L D+S)$, strength training $(S T)$, and strength training + steroid $(S T+S)$. (a) represents significant difference versus EA; (b) significant difference versus OLD; Among groups: $\mathrm{F}=7.832 ; p=0.0001$.

may directly influence the type of adjustment to be expected. Frontera et al ${ }^{24}$ showed that the capillary density decreases in the quadriceps femoris muscle over time. However, Chilibeck et al ${ }^{25}$ and Coggan et $\mathrm{al}^{26}$ demonstrated no significant change in the blood capillary density of the gastrocnemius muscle between young and elderly groups. However, Chilibeck et $\mathrm{al}^{25}$ also demonstrated that young and old groups had similar $\mathrm{VO}_{2}$ kinetics, muscle capillarization, and estimated $\mathrm{O}_{2}$ diffusion distances. Desaki et $\mathrm{al}^{27}$ suggested that capillaries from the fast-twitch extensor digitorum longus (EDL) muscle decreased over more advanced age. According to Desaki et $\mathrm{al}^{28}$ muscle capillarity can be reduced or even degenerate and/or be destroyed during advanced age. So far, the comparison between our data and the available literature demonstrated that, if the skeletal muscle capillarity profile were influenced by the aging process, it would just occur at very late ages in muscles with a predominance of fast-twitch myofibers. In addition, Muller-Delp et $\mathrm{al}^{29}$ investigated the hypothesis that aging results in increased vasoconstrictor responses of skeletal muscle resistance arterioles. The authors suggested that aging does not result in a nonspecific decrease in vasoconstrictor responsiveness of skeletal muscle arterioles. Rather, aging-induced adaptations of vasoreactivity of resistance arterioles appear to be limited to mechanisms that are uniquely involved in the signaling of the myogenic response.

Hagerman et $\mathrm{al}^{30}$ showed no significant difference in the blood capillary density of the vastus lateralis muscle of elderly people who trained to voluntary failure. However, Krause Neto et $\mathrm{al}^{11}$ demonstrated that ST may not influence vascularity adjustments in the fast-twitch EDL muscle. According to Chilibeck et al, ${ }^{25}$ capillarization and $\mathrm{VO}_{2}$ kinetics during exercise of a muscle group (lateral gastrocnemius) accustomed to everyday activity (walking) are well maintained in old individuals. Coggan et $\mathrm{al}^{26}$ explained that capillary density may be similar between young and old 
athletes because master athletes present 34\% larger type I fibers. Thus, the survival of the muscle fiber type present in the skeletal muscle determines the maintenance of the oxidative enzymatic profile and, consequently, the capillary-to-fiber ratio. ${ }^{26}$ However, the type of training might influence the expected adaptation. Dimauro et $\mathrm{al}^{31}$ studied the effects of high-intensity short-duration swimming training and anabolic steroid treatment on the medial gastrocnemius muscle of female rats. The authors showed that only swimming training but not anabolic steroid treatment resulted in significant increases in the number of capillaries surrounding type I and Ila myofibers. However, physiological dosages of androgenic anabolic steroids (AAS) may not be sufficient to induce muscle hypertrophy.

In the present study, we have demonstrated that testosterone administration influenced directly the adjustment of the vascular system in the capillary volume density of both groups treated with androgens. However, few studies investigated the effects of testosterone administration on the capillary density volume. Modulation of endothelial cell growth and function is a potential mechanism of androgen actions. Data from isolated vessels and animal models suggest that pharmacological doses of testosterone, or its potent intracellular metabolite dihydrotestosterone (DHT), produce vasodilation. ${ }^{32} \mathrm{Cai}_{\text {et }} \mathrm{l}^{33}$ demonstrated that androgens (DHT and testosterone) produced a time- and dose-dependent induction of cell proliferation in primary human aortic endothelial cells (HAECs), as evidenced by increases in viable cell number and DNA biosynthesis. Also, their study showed that DHT induced androgen receptor (AR), cyclin A and D1, and vascular endothelial growth factor (VEGF) gene expression in a dose- and time-dependent manner, indicating that the DHT effects are mediated via AR. Results of various studies suggest that testosterone can also alter vascular tone through both endothelium-dependent and endothelium-independent mechanisms in a variety of vascular beds and vessel types. ${ }^{32}$ In addition, the endotheliumdependent effects of testosterone are likely mediated at least in part through nitric oxide (NO) elaboration, whereas the mechanisms of endothelium-independent effects involve one or more types of smooth muscle ion conductance channels. These data explain, at least in part, the results obtained in the present study.

Skeletal muscle response might vary depending on the intervention applied and on the type of skeletal muscle analyzed. ${ }^{11}$ Kovacheva et al ${ }^{34}$ showed that cross-sectional area (CSA) from the gastrocnemius muscle increased in both fast-twitch muscle fibers after anabolic steroid administration by suppression of myostatin and enzyme c-Jun $\mathrm{N}$ terminal kinase (JNK). Nonetheless, it seems clear that hypertrophic response to anabolic steroid is dose-dependent, and that higher dosages of androgens might trigger greater muscle hypertrophy gains and capillary density increase. Griggs et $\mathrm{al}^{35}$ demonstrated that testosterone increases muscle mass by increasing protein synthesis. Strength training is also great to reduce the rates of muscle loss and increase muscle strength, being as effective as testosterone to increase the gene expression of myofibers of several growth factors and the synthesis of new proteins. ${ }^{36}$
Several studies demonstrated that a combination of ST and anabolic steroids were more effective to muscle hypertrophy than each intervention alone. ${ }^{37,38}$ This fact is explained by a more potent interaction with ARs after both treatments. ${ }^{39}$ However, we did not show a significant difference in muscle hypertrophy between the two groups. This fact can be explained by the training volumes (number of climbs + load by climbing), which were possibly similar in both groups. ${ }^{17}$

\section{Conclusion}

Although it is accepted that vascularity decreases during aging, the conflicting results found in the literature may be related to the sample number adopted in the research, to the type of intervention applied, to the different muscle groups used in each study, and also to the types of analyses adopted.

Through the results obtained in the present study, it was concluded that the administration of testosterone was crucial for the increase of capillary density and muscular hypertrophy of the groups with and without ST.

\section{Conflicts of Interests}

The authors have no conflicts of interests to declare.

\section{References}

1 Beaudart C, Locquet M, Reginster JY, Delandsheere L, Petermans J, Bruyère $O$. Quality of life in sarcopenia measured with the SarQoL®: impact of the use of different diagnosis definitions. Aging Clin Exp Res 2017;••• Doi: 10.1007/s40520-017-0866-9

2 Soares AV, Marcelino E, Maia KC, Borges NG Junior. Relação entre mobilidade funcional e dinapenia em idosos com fragilidade. Einstein (Sao Paulo) 2017;15(03):278-282

3 Nicholson CJ, Sweeney M, Robson SC, Taggart MJ. Estrogenic vascular effects are diminished by chronological aging. Sci Rep 2017;7(01):12153. Doi: 10.1038/s41598-017-12153-5

4 Xu X, Wang B, Ren C, et al. Recent Progress in Vascular Aging: Mechanisms and Its Role in Age-related Diseases. Aging Dis 2017; 8(04):486-505

5 Osuka Y, Fujita S, Kitano N, et al. Effects of Aerobic and Resistance Training Combined with Fortified Milk on Muscle Mass, Muscle Strength, and Physical Performance in Older Adults: A Randomized Controlled Trial. J Nutr Health Aging 2017;21(10):1349-1357. Doi: 10.1007/s12603-016-0864-1

6 Søgaard D, Lund MT, Scheuer CM, et al. High-intensity interval training improves insulin sensitivity in older individuals. Acta Physiol (Oxf) 2017;•••: [Epub ahead of print] . Doi: 10.1111/ apha.13009

7 Loyola WS, Camillo CA, Torres CV, Probst VS. Effects of an exercise model based on functional circuits in an older population with different levels of social participation. Geriatr Gerontol Int 2017; •.• Doi: 10.1111/ggi.13167

8 Holloszy JO, Coyle EF. Adaptations of skeletal muscle to endurance exercise and their metabolic consequences. J Appl Physiol 1984; 56(04):831-838

9 Prior BM, Yang HT, Terjung RL. What makes vessels grow with exercise training? J Appl Physiol (1985) 2004;97(03):1119-1128

10 Rossman MJ, Kaplon RE, Hill SD, et al. Endothelial cell senescence with aging in healthy humans: prevention by habitual exercise and relation to vascular endothelial function. Am J Physiol Heart Circ Physiol 2017; 313(05):H890-H895. Doi: 10.1152/ajpheart.00416.2017

11 Krause Neto W, Gama FE. Strength training and anabolic steroid do not affect muscle capillarization of middle-aged rats. Rev Bras Med Esporte 2017;23(02):137-141 
12 Hudlicka O, Brown M, Egginton S. Angiogenesis in skeletal and cardiac muscle. Physiol Rev 1992;72(02):369-417

13 Webb CM, Adamson DL, de Zeigler D, Collins P. Effect of acute testosterone on myocardial ischemia in men with coronary artery disease. Am J Cardiol 1999;83(03):437-439, A9

14 Hayward CS, Kelly RP, Collins P. The roles of gender, the menopause and hormone replacement on cardiovascular function. Cardiovasc Res 2000;46(01):28-49

15 Gonçalves L, de Souza RR, Maifrino LB, et al. Resistance exercise and testosterone treatment alters the proportion of numerical density of capillaries of the left ventricle of aging Wistar rats. Aging Male 2014;17(04):243-247

16 Deus APL, Bassi D, Simões RP, et al. MMP(-2) expression in skeletal muscle after strength training. Int J Sports Med 2012;33(02): 137-141

17 Krause Neto W, Silva WA, Ciena AP, et al. Total training load may explain similar strength gains and muscle hypertrophy seen in aged rats submitted to resistance training and anabolic steroids. Aging Male 2017; $\cdots$ :1-12. Doi: 10.1080/ 13685538.2017.1365832

18 Harris MB, Slack KN, Prestosa DT, Hryvniak DJ. Resistance training improves femoral artery endothelial dysfunction in aged rats. Eur J Appl Physiol 2010;108(03):533-540

19 Tanasov VS, Krause Neto W, Gonçalves L, Maifrino LBM, De Sousa RR, Gama EF. Use of Anabolic Steroid Altered the Liver Morphology of Rats. Int J Morphol 2014;32(03):756-760

20 Harris BA. The influence of endurance and resistance exercise on muscle capillarization in the elderly: a review. Acta Physiol Scand 2005;185(02):89-97

21 Hepple RT, Mathieu-Costello O. Estimating the size of the capillary-to-fiber interface in skeletal muscle: a comparison of methods. J Appl Physiol (1985) 2001;91(05):2150-2156

22 Andersen P. Capillary density in skeletal muscle of man. Acta Physiol Scand 1975;95(02):203-205

23 Degens H. Age-related changes in the microcirculation of skeletal muscle. Adv Exp Med Biol 1998;454:343-348

24 Frontera WR, Hughes VA, Fielding RA, Fiatarone MA, Evans WJ, Roubenoff R. Aging of skeletal muscle: a 12-yr longitudinal study. J Appl Physiol (1985) 2000;88(04):1321-1326

25 Chilibeck PD, Paterson DH, Cunningham DA, Taylor AW, Noble EG. Muscle capillarization 02 diffusion distance, and VO2 kinetics in old and young individuals. J Appl Physiol (1985) 1997;82(01): 63-69

26 Coggan AR, Spina RJ, Rogers MA, et al. Histochemical and enzymatic characteristics of skeletal muscle in master athletes. J Appl Physiol (1985) 1990;68(05):1896-1901
27 Desaki J, Nishida N. Structural changes of microvessels in the extensor digitorum longus muscle of the aged rat. J Electron Microsc (Tokyo) 2007;56(04):157-162

28 Desaki J, Oki S, Ezaki T. Remodelling of capillary networks around muscle fibres in the extensor digitorum longus muscle of the normal aged rat.J Electron Microsc (Tokyo) 2002;51(03):183-194

29 Muller-Delp Judy, Spier SA, Ramsey MW, et al. Effects of aging on vasoconstrictor and mechanical properties of rat skeletal muscle arterioles. Am J Physiol Heart Circ Physiol 2002;282:H1843-H1854 First published December 6, 2001. Doi: 10.1152/ ajpheart.00666.2001

30 Hagerman FC, Walsh SJ, Staron RS, et al. Effects of high-intensity resistance training on untrained older men. I. Strength, cardiovascular, and metabolic responses. J Gerontol A Biol Sci Med Sci 2000;55(07):B336-B346

31 Dimauro J, Balnave RJ, Shorey CD. Effects of anabolic steroids and high intensity exercise on rat skeletal muscle fibres and capillarization. A morphometric study. Eur J Appl Physiol Occup Physiol 1992;64(03):204-212

32 Littleton-Kearney M, Hurn PD. Testosterone as a modulator of vascular behavior. Biol Res Nurs 2004;5(04):276-285. Doi: $10.1177 / 1099800403262927$

33 Cai J, Hong Y, Weng C, Tan C, Imperato-McGinley J, Zhu Y-S. Androgen stimulates endothelial cell proliferation via an androgen receptor/VEGF/cyclin A-mediated mechanism. Am J Physiol Heart Circ Physiol 2011;300(04):H1210-H1221. Doi: 10.1152/ ajpheart.01210.2010

34 Kovacheva EL, Hikim AP, Shen R, Sinha I, Sinha-Hikim I. Testosterone supplementation reverses sarcopenia in aging through regulation of myostatin, c-Jun NH2-terminal kinase, Notch, and Akt signaling pathways. Endocrinology 2010;151(02):628-638

35 Griggs RC, Kingston W, Jozefowicz RF, Herr BE, Forbes G, Halliday D. Effect of testosterone on muscle mass and muscle protein synthesis. J Appl Physiol (1985) 1989;66(01):498-503

36 Snijders T, Verdijk LB, van Loon LJ. The impact of sarcopenia and exercise training on skeletal muscle satellite cells. Ageing Res Rev 2009;8(04):328-338

37 Bhasin S, Storer TW, Berman N, et al. The effects of supraphysiologic doses of testosterone on muscle size and strength in normal men. N Engl J Med 1996;335(01):1-7

38 Bhasin S, Storer TW, Javanbakht M, et al. Testosterone replacement and resistance exercise in HIV-infected men with weight loss and low testosterone levels. JAMA 2000;283(06):763-770

39 Willoughby DS, Taylor L. Effects of sequential bouts of resistance exercise on androgen receptor expression. Med Sci Sports Exerc 2004;36(09):1499-1506 